Annals of Plant Sciences

ISSN: 2287-688X

OPEN ACCESS

www.annalsofplantsciences.com

Research Article

\title{
Checklist of Akshara vidyalaya campus flora, Venkatachalam, Nellore, Andhra Pradesh, India.
}

Manipal K. ${ }^{*}$, Kumuda M.V. ${ }^{2}$, Madhava Chetty K. ${ }^{1}$

${ }^{1}$ Department of Botany, Sri Venkateswara University, Tirupati, Chittoor Dt. - 517502, A.P., India

2Department of Biological sciences, Akshara Vidyalaya, Venkatachalam, SPSR Nellore Dt- 524320, A.P., India.

Received: 06-02-2018; Revised: 22-07-2018; Accepted: 02-8-2018

\begin{abstract}
The present study was aimed at determining the plant species richness of the Akshara Vidyalaya (AV) campus, Nellore District of Andhra pradesh. For this, the species richness data was obtained by intensive surveys in the campus from 2015-2017. We recorded 247 species in 194 genera belonging to 69 families composed of 52 trees, 55 shrubs and 140 herbs, two species are parasites. Poaceae (20 genera 24 species), Fabaceae (15 genera 21 species) are the two species richness families and an inventory checklist of all the species recorded is provided. This study reveals that AV campus is diverse and rich in native, ornamental and alien flora. We recommend educational institutions to develop Botanical garden with the medicinal and economical plant taxa of wild varities for educating students and exploring the interconnections with the biota to environment.
\end{abstract}

Keywords: Akshara vidyalaya campus, floristic studies, Checklist.

\section{Introduction}

Educational institutions as curricula should introduce students to the incredible biodiversity of flora, and the connections between plants and their ecosystems. Fundamental floristic knowledge is one of the priority actions for any student in educating about biodiversity and living world. In recent years, comprehensive floristic accounts have been published for various areas with micro and macro habitats. Akshara Vidyalaya (AV), an international school run by Swarna Bharat Trust at Venkatachalam in SPSR Nellore district comprises student training in different activities in biology and its allied aspects. As a part of the institutional curricula, the present work has been undertaken which deals with the enumeration and catalouge the diversity of plants habituating in AV Campus. This study is an attempt to identify and classify the plants using basic biological criteria with recording the flora of the Akshara Vidyalaya.

\section{Materials and Methods}

\section{Study area}

This study was conducted in 26 acre land of Akshara Vidyalaya. Climatic conditions occur as Southwest Monsoon from June to September, Northeast Monsoon from October to December. Temperature ranges Maximum $36^{\circ} \mathrm{C}$ to $46^{\circ} \mathrm{C}$ (During Summer), Minimum $23^{\circ} \mathrm{C}$ to $25^{\circ} \mathrm{c}$ (During Winter), Rainfall ranges from $700-1000 \mathrm{~mm}$ from Southwest and Northeast monsoons Topography of AV campus

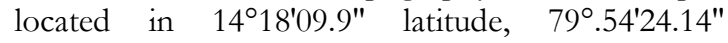
longitude (GPS) (Map.1).

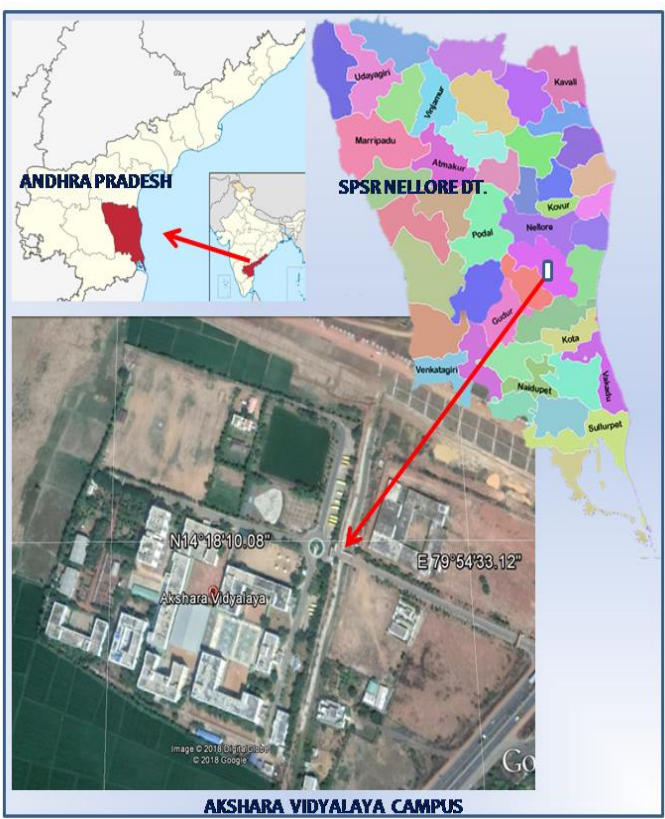

Map 1. Location map of AV Campus

\section{Data collection}

The entire work was undertaken from August 2015 to June 2017. Bentham and Hooker's Natural system of classification was followed to classify the species. Author citation and binomial of collected species were verified with authentic databases such as International Plant Names Index (IPNI) and The Plant list (2010). All specimens collected were identified with regional floras (Gamble and Fischer, 1957; Suryanarayana and Sreenivasa Rao, 2002, Pullaiah et al.,1997; Pullaiah,1998; Madhava Chetty

\footnotetext{
*Corresponding Author:

Manipal K., BSR-SRF,

Department of Botany,

Sri Venkateswara University, Tirupati,

Chittoor Dt. - 517502, A.P., India.

E-mail: drmanipalbotany@gmail.com
}

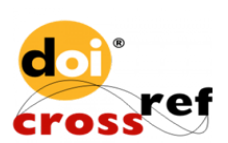


et al., 2015) and also from experts (acknowledged). The collected specimens were compared with the herbarium specimens housed at Department of Botany, Sri Venkateswara university, Tirupati (SVUTY). The voucher specimens were tagged a code (AVN/Family Abb/Specimen Number) for further reference.

\section{Results and Discussion}

The floristic enumeration of Akshara Vidyalaya, Venkatachalam, Nellore, reports a total of 247 species in 194 genera belonging to 69 families. The detailed inventory of the species is provided in Table 1 name of the identified plants, family, Lifeform and Voucher specimen code. some species are depicted in Images (Fig.2, A-T). Out of 247 species, 42 species in 33 genera belongs to monocotyledons, 205 species in 158 genera belongs to dicotyledons. Among the species recorded
Poaceae with 20 genera 24 species (Monocotyledons), Fabaceae with 15 genera 21 species (Dicotyledons) are the two species richness families in AV Campus. Of the 250 taxa 53 are trees, 56 are shrubs, and 140 are herbs, Cuscuta reflexa Roxb., and Striga angustifolia (D.Don) C.J. Saldanha are the parasites reported. $60 \%$ of the plant species reported are medicinally important. 50 $\%$ of the species are aliens only $20 \%$ are native species of India. The life-form composition analysis shows that trees are dominant thanshrubs and herbs. No uncommon or rare species reported. AV authorities made efforts to establish horticultural/ornamental varities in the Garden and campus surroundings. However, composition of the AV flora consists of nearly an higher percentage of exotic and very lower percentage of native species. For the Greenery of the Campus many ornamentals were planted for landscaping and beautification.

Table 1. Checklist of Flora of Akshara Vidyalaya (AV) Campus, Nellore District, Andhra Pradesh.

\begin{tabular}{|c|c|c|c|c|}
\hline \multicolumn{5}{|c|}{ DICOTYLEDONS } \\
\hline S.No & Name of the Plant & Family & Life form & Voucher Number \\
\hline 1. & Dillenia indica L. & Dilleniaceae & $\mathrm{T}$ & AVN/DIL/001 \\
\hline 2. & Michelia champaca L. & Magnoliaceae & $\mathrm{T}$ & $\mathrm{AVN} / \mathrm{MAG} / 002$ \\
\hline 3. & Annona reticulata $\mathrm{L}$. & Annonaceae & $\mathrm{S}$ & $\mathrm{AVN} / \mathrm{ANN} / 003$ \\
\hline 4. & Annona squamosa $\mathrm{L}$. & Annonaceae & $\mathrm{T}$ & $\mathrm{AVN} / \mathrm{ANN} / 0004$ \\
\hline 5. & Artabotrys hexapetalus (L.f.) Bhandari & Annonaceae & $\mathrm{S}$ & AVN/ANN/0005 \\
\hline 6. & Polyalthia longifolia (Sonn.) Thwaites & Annonaceae & $\mathrm{T}$ & AVN/ANN/0006 \\
\hline 7. & Cocculus hirsutus (L.) W.Theob. & Menispermaceae & $\mathrm{C}$ & AVN/MEN/0007 \\
\hline 8. & Tinospora sinensis (Lour.) Merr. & Menispermaceae & $\mathrm{C}$ & AVN/MEN/0008 \\
\hline 9. & Capparis zeylanica $\mathrm{L}$. & Capparaceae & $\mathrm{C}$ & AVN/CAP/0009 \\
\hline 10. & Cleome viscosa $\mathrm{L}$. & Cleomaceae & $\mathrm{H}$ & AVN/CLE/0010 \\
\hline 11. & Hybanthus enneaspermus (L.) F.Muell. & Violaceae & $\mathrm{H}$ & AVN/VIO/0011 \\
\hline 12. & Polygala arvensis Willd. & Polygalaceae & $\mathrm{H}$ & AVN/POL/0012 \\
\hline 13. & Portulaca grandiflora Hook. & Portulacaceae & $\mathrm{H}$ & AVN/POR/0013 \\
\hline 14. & Portulaca oleracea $\mathrm{L}$. & Portulacaceae & $\mathrm{H}$ & AVN/POR/0014 \\
\hline 15. & Portulaca quadrifida $\mathrm{L}$. & Portulacaceae & $\mathrm{H}$ & AVN/POR/0015 \\
\hline 16. & Abutilon indicum (L.) Sweet & Malvaceae & $\mathrm{S}$ & AVN/MAL/0016 \\
\hline 17. & Hibiscus micranthus L.f. & Malvaceae & $\mathrm{H}$ & AVN/MAL/0017 \\
\hline 18. & Hibiscus rosa-sinensis $\mathrm{L}$. & Malvaceae & $\mathrm{S}$ & AVN/MAL/0018 \\
\hline 19. & Malvastrum coromandelianum (L.) Garcke & Malvaceae & $\mathrm{H}$ & AVN/MAL/0019 \\
\hline 20. & Sida acuta Burm.f. & Malvaceae & $\mathrm{H}$ & AVN/MAL/0020 \\
\hline 21. & Sida cordata (Burm.f.) Borss.Waalk. & Malvaceae & $\mathrm{H}$ & AVN/MAL/0021 \\
\hline 22. & Sterculia foetida $\mathrm{L}$. & Sterculiaceae & $\mathrm{T}$ & AVN/STE/0022 \\
\hline 23. & Melochia corchorifolia $\mathrm{L}$. & Sterculiaceae & $\mathrm{H}$ & AVN/STE/0023 \\
\hline 24. & Corchorus trilocularis L. & Tiliaceae & $\mathrm{H}$ & AVN/TIL/0024 \\
\hline 25. & Triumfetta rotundifolia Lam. & Tiliaceae & $\mathrm{H}$ & AVN/TIL/0025 \\
\hline 26. & Muntingia calabura L. & Elaeocarpaceae & $\mathrm{T}$ & AVN/ELA/0026 \\
\hline 27. & Tribulus terrestris $\mathrm{L}$. & Zygophyllaceae & $\mathrm{H}$ & AVN/ZYG/0027 \\
\hline 28. & Aegle marmelos (L.) Corrêa & Rutaceae & $\mathrm{T}$ & AVN/RUT/0028 \\
\hline 29. & Citrus grandis (L.) Osbeck & Rutaceae & $\mathrm{T}$ & AVN/RUT/0029 \\
\hline 30. & Citrus limon (L.) Osbeck & Rutaceae & $\mathrm{T}$ & AVN/RUT/0030 \\
\hline 31. & Murraya koenigii (L.) Spreng. & Rutaceae & $\mathrm{T}$ & AVN/RUT/0031 \\
\hline 32. & Azadirachta indica A.Juss. & Meliaceae & $\mathrm{T}$ & AVN/MEL/0032 \\
\hline 33. & Ziziphus jujuba Mill. & Rhamnaceae & $\mathrm{T}$ & AVN/RHA/0033 \\
\hline 34. & Cissus vitiginea $\mathrm{L}$. & Vitaceae & $\mathrm{S}$ & AVN/VIT/0034 \\
\hline 35. & Anacardium occidentale L. & Anacardiacae & $\mathrm{T}$ & AVN/ANA/0035 \\
\hline 36. & Mangifera indica $\mathrm{L}$. & Anacardiacae & $\mathrm{T}$ & AVN/ANA/0036 \\
\hline 37. & Moringa oleifera Lam. & Moringaceae & $\mathrm{T}$ & AVN/MOR/0037 \\
\hline 38. & Aeschynomene aspera Willd. & Fabaceae & $\mathrm{S}$ & AVN/FAB/0038 \\
\hline 39. & Alysicarpus monilifer (L.) DC. & Fabaceae & $\mathrm{H}$ & AVN/FAB/0039 \\
\hline 40. & Butea monosperma (Lam.) Taub. & Fabaceae & $\mathrm{T}$ & AVN/FAB/0040 \\
\hline 41. & Clitoria ternatea $\mathrm{L}$. & Fabaceae & $\mathrm{C}$ & AVN/FAB/0041 \\
\hline 42. & Crotalaria angulata Mill. & Fabaceae & $\mathrm{H}$ & AVN/FAB/0042 \\
\hline 43. & Crotalaria hebecarpa (DC.) Rudd & Fabaceae & $\mathrm{H}$ & AVN/FAB/0043 \\
\hline 44. & Crotalaria medicaginea var. neglecta (Wight \& Arn.) Baker & Fabaceae & $\mathrm{H}$ & $\mathrm{AVN} / \mathrm{FAB} / 0044$ \\
\hline 45. & Crotalaria verrucosa $\mathrm{L}$. & Fabaceae & $\mathrm{H}$ & $\mathrm{AVN} / \mathrm{FAB} / 0045$ \\
\hline
\end{tabular}




\begin{tabular}{|c|c|c|c|c|}
\hline 46. & Desmodium triflorum (L.) DC & Fabaceae & $\mathrm{H}$ & AVN/FAB/0046 \\
\hline 47. & Galactia tenuiflora (Willd.) Wight \& Arn. & Fabaceae & $\mathrm{H}$ & $\mathrm{AVN} / \mathrm{FAB} / 0047$ \\
\hline 48. & Gliricidia sepium (Jacq.) Walp. & Fabaceae & $\mathrm{T}$ & AVN/FAB/0048 \\
\hline 49. & Indigofera linifolia (L.f.) Retz. & Fabaceae & $\mathrm{H}$ & $\mathrm{AVN} / \mathrm{FAB} / 0049$ \\
\hline 50. & Indigofera linnaei Ali & Fabaceae & $\mathrm{H}$ & AVN/FAB/0050 \\
\hline 51. & Pongamia pinnata (L.) Pierre & Fabaceae & $\mathrm{T}$ & AVN/FAB/0051 \\
\hline 52. & Pterocarpus marsupium Roxb. & Fabaceae & $\mathrm{T}$ & AVN/FAB/0052 \\
\hline 53. & Pterocarpus santalinus L.f. & Fabaceae & $\mathrm{T}$ & AVN/FAB/0053 \\
\hline 54. & Rhynchosia minima (L.) DC. & Fabaceae & $\mathrm{C}$ & AVN/FAB/0054 \\
\hline 55. & Sesbania sesban (L.) Merr. & Fabaceae & $\mathrm{T}$ & AVN/FAB/0055 \\
\hline 56. & Tephrosia purpurea (L.) Pers. & Fabaceae & $\mathrm{H}$ & AVN/FAB/0056 \\
\hline 57. & Tephrosia procumbens (Ham.) Benth. & Fabaceae & $\mathrm{H}$ & AVN/FAB/0057 \\
\hline 58. & Vigna trilobata (L.) Verdc. & Fabaceae & $\mathrm{H}$ & AVN/FAB/0058 \\
\hline 59. & Baubinia purpurea L. & Caesalpiniaceae & $\mathrm{T}$ & AVN/CAE/0059 \\
\hline 60. & Caesalpinia pulcherrima (L.) Sw. & Caesalpiniaceae & $\mathrm{S}$ & $\mathrm{AVN} / \mathrm{CAE} / 0060$ \\
\hline 61. & Peltophorum pterocarpum (DC.) K.Heyne & Caesalpiniaceae & $\mathrm{T}$ & AVN/CAE/0061 \\
\hline 62. & Saraca asoca (Roxb.) Willd. & Caesalpiniaceae & $\mathrm{T}$ & AVN/CAE/0062 \\
\hline 63. & Tamarindus indica $\mathrm{L}$. & Caesalpiniaceae & $\mathrm{T}$ & AVN/CAE/0063 \\
\hline 64. & Mimosa pudica L. & Mimosaceae & $\mathrm{H}$ & AVN/MIM/0064 \\
\hline 65. & Pithecellobium dulce (Roxb.) Benth. & Mimosaceae & $\mathrm{T}$ & $\mathrm{AVN} / \mathrm{MIM} / 0065$ \\
\hline 66. & Rosa centifolia $\mathrm{L}$. & Rosaceae & $\mathrm{s}$ & AVN/ROS/0066 \\
\hline 67. & Combretum indicum (L.) DeFilipps & Combretaceae & S & $\mathrm{AVN} / \mathrm{COM} / 0067$ \\
\hline 68. & Bucida molinetii (M.Gómez) Alwan \& Stace & Combretaceae & $\mathrm{T}$ & $\mathrm{AVN} / \mathrm{COM} / 0068$ \\
\hline 69. & Psidium guajava $\mathrm{L}$. & Myrtaceae & $\mathrm{T}$ & AVN/MYR/0069 \\
\hline 70. & Sysygium cumini (L.) Skeels & Myrtaceae & $\mathrm{T}$ & $\mathrm{AVN} / \mathrm{MYR} / 0070$ \\
\hline 71. & Ammannia baccifera $\mathrm{L}$. & Lythraceae & $\mathrm{H}$ & AVN/LYT/0071 \\
\hline 72. & Lawsonia inermis $\mathrm{L}$. & Lythraceae & S & AVN/LYT/0072 \\
\hline 73. & Punica granatum $\mathrm{L}$. & Punicaceae & $\mathrm{S}$ & AVN/PUN/0073 \\
\hline 74. & Ludwigia perennis L. & Onagraceae & $\mathrm{H}$ & AVN/ONA/0074 \\
\hline 75. & Passiflora foetida $\mathrm{L}$. & Passifloraceae & $\mathrm{C}$ & AVN/PAS/0075 \\
\hline 76. & Passiflora alata Curtis & Passifloraceae & $\mathrm{C}$ & AVN/PAS/0076 \\
\hline 77. & Passiflora incarnata $\mathrm{L}$. & Passifloraceae & $\mathrm{C}$ & AVN/PAS/0077 \\
\hline 78. & Citrullus colocynthis (L.) Schrad. & Cucurbitaceae & $\mathrm{C}$ & AVN/CUC/0078 \\
\hline 79. & Mukia maderaspatana (L.) M.Roem. & Cucurbitaceae & $\mathrm{C}$ & $\mathrm{AVN} / \mathrm{CUC} / 0079$ \\
\hline 80. & Opuntia dillenii (Ker Gawl.) Haw. & Cactaceae & $\mathrm{S}$ & AVN/CAC/0080 \\
\hline 81. & Trianthema portulacastrum $\mathrm{L}$. & Aizoaceae & $\mathrm{H}$ & AVN/AIZ/0081 \\
\hline 82. & Glinus lotoides L. & Molluginaceae & $\mathrm{H}$ & AVN/MOL/0082 \\
\hline 83. & Mollugo oppositifolia L. & Molluginaceae & $\mathrm{H}$ & AVN/MOL/0083 \\
\hline 84. & Mollugo nudicaulis Lam. & Molluginaceae & $\mathrm{H}$ & AVN/MOL/0084 \\
\hline 85. & Mollugo pentaphylla $\mathrm{L}$. & Molluginaceae & $\mathrm{H}$ & AVN/MOL/0085 \\
\hline 86. & Dentella repens (L.) J.R.Forst. \& G.Forst. & Rubiaceae & $\mathrm{H}$ & $\mathrm{AVN} / \mathrm{RUB} / 0086$ \\
\hline 87. & Ixora coccinea $\mathrm{L}$. & Rubiaceae & $\mathrm{S}$ & AVN/RUB/0087 \\
\hline 88. & Ixora chinensis Lam. & Rubiaceae & S & AVN/RUB/0088 \\
\hline 89. & Neolamarckia cadamba (Roxb.) Bosser & Rubiaceae & $\mathrm{T}$ & AVN/RUB/0089 \\
\hline 90. & Spermacoce hispida $\mathrm{L}$. & Rubiaceae & $\mathrm{H}$ & AVN/RUB/0090 \\
\hline 91. & Oldenlandia umbellata $\mathrm{L}$. & Rubiaceae & $\mathrm{H}$ & AVN/RUB/0091 \\
\hline 92. & Oldenlandia corymbosa $\mathrm{L}$. & Rubiaceae & $\mathrm{H}$ & AVN/RUB/0092 \\
\hline 93. & Catunaregam spinosa (Thunb.) Tirveng. & Rubiaceae & $\mathrm{H}$ & AVN/RUB/0093 \\
\hline 94. & Thecagonum pteritum (Blume) Babu & Rubiaceae & $\mathrm{H}$ & AVN/RUB/0094 \\
\hline 95. & Mussaenda philippica A.Rich. & Rubiaceae & $\mathrm{S}$ & AVN/RUB/0095 \\
\hline 96. & Ageratum conyzoides (L.) L. & Asteraceae & $\mathrm{H}$ & AVN/AST/0096 \\
\hline 97. & Eclipta prostrata (L.) L. & Asteraceae & $\mathrm{H}$ & AVN/AST/0097 \\
\hline 98. & Emilia sonchifolia (L.) DC. ex DC. & Asteraceae & $\mathrm{H}$ & AVN/AST/0098 \\
\hline 99. & Tridax procumbens (L.) L. & Asteraceae & $\mathrm{H}$ & AVN/AST/0099 \\
\hline 100. & Vernonia albicans DC. & Asteraceae & $\mathrm{H}$ & AVN/AST/0100 \\
\hline 101. & Vernonia cinerea (L.) Less. & Asteraceae & $\mathrm{H}$ & AVN/AST/0101 \\
\hline 102. & Tricholepis radicans (Roxb.) DC. & Asteraceae & $\mathrm{H}$ & AVN/AST/0102 \\
\hline 103. & Manilkara zapota (L.) P.Royen & Sapotaceae & $\mathrm{T}$ & AVN/SAP/0103 \\
\hline 104. & Mimusops elengi $\mathrm{L}$. & Sapotaceae & $\mathrm{T}$ & AVN/SAP/0104 \\
\hline 105. & Jasminum calophyllum Wall. \& G.Don & Oleaceae & $\mathrm{C}$ & AVN/OLE/0105 \\
\hline 106. & Nyctanthes arbor-tristis $\mathrm{L}$. & Oleaceae & $\mathrm{S}$ & AVN/OLE/0106 \\
\hline 107. & Adenium obesum (Forssk.) Roem. \& Schult. & Apocynaceae & $\mathrm{S}$ & $\mathrm{AVN} / \mathrm{APO} / 0107$ \\
\hline 108. & Allamanda blanchetii A.DC. & Apocynaceae & $\mathrm{S}$ & AVN/APO/0108 \\
\hline 109. & Allamanda cathartica $\mathrm{L}$. & Apocynaceae & $\mathrm{T}$ & $\mathrm{AVN} / \mathrm{APO} / 0109$ \\
\hline 110. & Alstonia scholaris (L.) R. Br. & Apocynaceae & S & AVN/APO/0110 \\
\hline 111. & Carissa spinarum $\mathrm{L}$. & Apocynaceae & $\mathrm{H}$ & AVN/APO/0111 \\
\hline 112. & Catharanthus roseus (L.) G.Don & Apocynaceae & S & AVN/APO/0112 \\
\hline 113. & Nerium oleander $\mathrm{L}$. & Apocynaceae & $\mathrm{S}$ & AVN/APO/0113 \\
\hline 114. & Plumeria alba $\mathrm{L}$. & Apocynaceae & $\mathrm{T}$ & $\mathrm{AVN} / \mathrm{APO} / 0114$ \\
\hline 115. & Plumeria rubra L. & Apocynaceae & $\mathrm{T}$ & $\mathrm{AVN} / \mathrm{APO} / 0115$ \\
\hline 116. & Plumeria obtusa L. & Apocynaceae & $\mathrm{S}$ & $\mathrm{AVN} / \mathrm{APO} / 0116$ \\
\hline 117. & Plumeria pudica Jacq. & Apocynaceae & $\mathrm{S}$ & $\mathrm{AVN} / \mathrm{APO} / 0117$ \\
\hline
\end{tabular}




\begin{tabular}{|c|c|c|c|c|}
\hline 118. & Tabernaemontana divaricata (L.) R.Br. ex Roem. \& Schult. & Apocynaceae & $\mathrm{S}$ & $\mathrm{AVN} / \mathrm{APO} / 0118$ \\
\hline 119. & Calotropis procera (Aiton) Dryand. & Asclepiadaceae & $\mathrm{C}$ & AVN/ASC/0119 \\
\hline 120. & Pentatropis capensis (L. f.) Bullock & Asclepiadaceae & $\mathrm{C}$ & AVN/ASC/0120 \\
\hline 121. & Pergularia daemia (Forssk.) Chiov. & Asclepiadaceae & $\mathrm{C}$ & AVN/ASC/0121 \\
\hline 122. & Oxystelma esculentum (L. f.) Sm. & Asclepiadaceae & $\mathrm{C}$ & AVN/ASC/0122 \\
\hline 123. & Wattakaka volubilis (L. f.) Stapf & Asclepiadaceae & $\mathrm{C}$ & AVN/ASC/0123 \\
\hline 124. & Hemidesmus indicus (L.) R. Br. ex Schult. & Periplocaceae & $\mathrm{C}$ & AVN/PER/0124 \\
\hline 125. & Hemidesmus indicus var. pubescens Hook.. & Periplocaceae & $\mathrm{C}$ & AVN/PER/0125 \\
\hline 126. & Hydrolea zeylanica (L.) Vahl & Hydrophyllaceae & $\mathrm{H}$ & AVN/HYD/0126 \\
\hline 127. & Coldenia procumbens L. & Boraginaceae & $\mathrm{H}$ & AVN/BOR/0127 \\
\hline 128. & Trichodesma indicum (L.) Lehm. & Boraginaceae & $\mathrm{H}$ & AVN/BOR/0128 \\
\hline 129. & Heliotropium indicum $\mathrm{L}$. & Boraginaceae & $\mathrm{H}$ & AVN/BOR/0129 \\
\hline 130. & Evolvulus alsinoides (L.) L. & Convolvulaceae & $\mathrm{H}$ & $\mathrm{AVN} / \mathrm{CON} / 0130$ \\
\hline 131. & Evolvulus nummularius (L.) L. & Convolvulaceae & $\mathrm{H}$ & AVN/CON/0131 \\
\hline 132. & Ipomoea aquatica Forssk. & Convolvulaceae & $\mathrm{C}$ & AVN/CON/0132 \\
\hline 133. & Ipomoea marginata (Desr.) Verdc. & Convolvulaceae & $\mathrm{C}$ & AVN/CON/0133 \\
\hline 134. & Ipomoea quamoclit $\mathrm{L}$. & Convolvulaceae & $\mathrm{C}$ & AVN/CON/0134 \\
\hline 135. & Merremia tridentata (L.) Hallier $\mathrm{f}$. & Convolvulaceae & $\mathrm{C}$ & AVN/CON/0135 \\
\hline 136. & Merremia emarginata (Burm. f.) Hallier f. & Convolvulaceae & $\mathrm{C}$ & AVN/CON/0136 \\
\hline 137. & Rivea hypocrateriformis Choisy & Convolvulaceae & $\mathrm{C}$ & AVN/CON/0137 \\
\hline 138. & Cuscuta reflexa Roxb. & Convolvulaceae & $\mathrm{C}$ & AVN/CON/0138 \\
\hline 139. & Physalis minima $\mathrm{L}$. & Solanaceae & $\mathrm{H}$ & AVN/SOL/0139 \\
\hline 140. & Solanum surattense Burm. f. & Solanaceae & $\mathrm{H}$ & AVN/SOL/0140 \\
\hline 141. & Scoparia dulcis $\mathrm{L}$. & Scrophulariaceae & $\mathrm{H}$ & AVN/SCR/0141 \\
\hline 142. & Striga angustifolia (D. Don) C.J. Saldanha & Scrophulariaceae & $\mathrm{E}$ & AVN/SCR/0142 \\
\hline 143. & Millingtonia bortensis L.f. & Bignoniaceae & $\mathrm{T}$ & AVN/BIG/0143 \\
\hline 144. & Spathodea campanulata P.Beauv. & Bignoniaceae & $\mathrm{T}$ & AVN/BIG/0144 \\
\hline 145. & Tecoma stans (L.) Juss. ex Kunth & Bignoniaceae & $\mathrm{S}$ & AVN/BIG/0145 \\
\hline 146. & Tabebuia dubia (C.Wright ex Sauvalle) Britton ex Seibert & Bignoniaceae & $\mathrm{T}$ & AVN/BIG/0146 \\
\hline 147. & Asystasia gangetica (L.) T.Anderson & Acanthaceae & $\mathrm{H}$ & AVN/ACA/0147 \\
\hline 148. & Barleria prionitis $\mathrm{L}$. & Acanthaceae & $\mathrm{H}$ & AVN/ACA/0148 \\
\hline 149. & Crossandra infundibuliformis (L.) Nees & Acanthaceae & $\mathrm{H}$ & AVN/ACA/0149 \\
\hline 150. & Ecbolium viride (Forssk.) Alston & Acanthaceae & $\mathrm{H}$ & AVN/ACA/0150 \\
\hline 151. & Justicia adhatoda $\mathrm{L}$. & Acanthaceae & $\mathrm{S}$ & AVN/ACA/0151 \\
\hline 152. & Justicia prostrata Gamble & Acanthaceae & $\mathrm{H}$ & AVN/ACA/0152 \\
\hline 153. & Lepidagathis cristata Willd. & Acanthaceae & $\mathrm{H}$ & AVN/ACA/0153 \\
\hline 154. & Rungia repens (L.) Nees & Acanthaceae & $\mathrm{H}$ & AVN/ACA/0154 \\
\hline 155. & Andrographis echioides (L.) Nees & Acanthaceae & $\mathrm{H}$ & AVN/ACA/0155 \\
\hline 156. & Clerodendrum inerme (L.) Gaertn. & Verbenaceae & $\mathrm{S}$ & AVN/VER/0156 \\
\hline 157. & Duranta repens $\mathrm{L}$. & Verbenaceae & $\mathrm{H}$ & AVN/VER/0157 \\
\hline 158. & Phyla nodiflora (L.) Greene & Verbenaceae & $\mathrm{S}$ & AVN/VER/0158 \\
\hline 159. & Vitex negundo L. & Verbenaceae & $\mathrm{S}$ & AVN/VER/0159 \\
\hline 160. & Stachytarpheta jamaicensis (L.) Vahl & Verbenaceae & $\mathrm{H}$ & AVN/VER/0160 \\
\hline 161. & Tectona grandis L.f. & Verbenaceae & $\mathrm{T}$ & AVN/VER/0161 \\
\hline 162. & Anisomeles indica (L.) Kuntze & Lamiaceae & $\mathrm{S}$ & AVN/LAM/0162 \\
\hline 163. & Hyptis suaveolens (L.) Poit. & Lamiaceae & $\mathrm{S}$ & AVN/LAM/0163 \\
\hline 164. & Ocimum gratissimum $\mathrm{L}$. & Lamiaceae & $\mathrm{S}$ & AVN/LAM/0164 \\
\hline 165. & Ocimum tenuiflorum $\mathrm{L}$. & Lamiaceae & S & AVN/LAM/0165 \\
\hline 166. & Ocimum basilicum $\mathrm{L}$. & Lamiaceae & $\mathrm{H}$ & AVN/LAM/0166 \\
\hline 167. & Plectranthus amboinicus (Lour.) Spreng. & Lamiaceae & $\mathrm{H}$ & AVN/LAM/0167 \\
\hline 168. & Boerbavia erecta $\mathrm{L}$. & Nyctaginaceae & $\mathrm{H}$ & AVN/NYC/0168 \\
\hline 169. & Boerhavia diffusa $\mathrm{L}$. & Nyctaginaceae & $\mathrm{H}$ & AVN/NYC/0169 \\
\hline 170. & Bougainvillea spectabilis Willd. & Nyctaginaceae & $\mathrm{S}$ & AVN/NYC/0170 \\
\hline 171. & Achyranthes aspera $\mathrm{L}$. & Amaranthaceae & $\mathrm{H}$ & AVN/AMA/0171 \\
\hline 172. & Aerva lanata (L.) Juss. & Amaranthaceae & $\mathrm{H}$ & AVN/AMA/0172 \\
\hline 173. & Alternanthera tenella Colla & Amaranthaceae & $\mathrm{H}$ & AVN/AMA/0173 \\
\hline 174. & Amaranthus viridis $\mathrm{L}$. & Amaranthaceae & $\mathrm{H}$ & AVN/AMA/0174 \\
\hline 175. & Celosia argentea $\mathrm{L}$. var. argentea & Amaranthaceae & $\mathrm{H}$ & AVN/AMA/0175 \\
\hline 176. & Celosia argentea $\mathrm{f}$. cristata (L.) Schinz & Amaranthaceae & $\mathrm{H}$ & AVN/AMA/0176 \\
\hline 177. & Celosia argentea $\mathrm{L}$. var. plumosa & Amaranthaceae & $\mathrm{H}$ & AVN/AMA/0177 \\
\hline 178. & Gomphrena globosa L. & Amaranthaceae & $\mathrm{H}$ & AVN/AMA/0178 \\
\hline 179. & Gomphrena serrata $\mathrm{L}$. & Amaranthaceae & $\mathrm{H}$ & AVN/AMA/0179 \\
\hline 180. & Digera muricata (L.) Mart. & Amaranthaceae & $\mathrm{H}$ & AVN/AMA/0180 \\
\hline 181. & Pupalia lappacea (L.) Juss. & Amaranthaceae & $\mathrm{H}$ & AVN/AMA/0181 \\
\hline 182. & Tricburiella monsoniae (L. f.) Bennet & Amaranthaceae & $\mathrm{H}$ & AVN/AMA/0182 \\
\hline 183. & Grevillea robusta A.Cunn. ex R.Br. & Proteaceae & $\mathrm{T}$ & AVN/PRO/0183 \\
\hline 184. & Acalypha indica $\mathrm{L}$. & Euphorbiaceae & $\mathrm{H}$ & AVN/EUP/0184 \\
\hline 185. & Acalypha ciliata Wall. & Euphorbiaceae & $\mathrm{H}$ & AVN/EUP/0185 \\
\hline 186. & Acalypha wilkesiana Müll.-Arg. & Euphorbiaceae & $\mathrm{H}$ & AVN/EUP/0186 \\
\hline 187. & Euphorbia hirta L. & Euphorbiaceae & $\mathrm{H}$ & AVN/EUP/0187 \\
\hline 188. & Euphorbia hypericifolia L. & Euphorbiaceae & $\mathrm{H}$ & AVN/EUP/0188 \\
\hline 189. & Euphorbia heterophylla L. & Euphorbiaceae & $\mathrm{H}$ & AVN/EUP/0189 \\
\hline
\end{tabular}




\begin{tabular}{|c|c|c|c|c|}
\hline & & & & \\
\hline 190. & Euphorbia milii Des Moul. & Euphorbiaceae & $\mathrm{H}$ & AVN/EUP/0190 \\
\hline 191. & Euphorbia serpens Kunth & Euphorbiaceae & $\mathrm{H}$ & AVN/EUP/0191 \\
\hline 192. & Euphorbia clarkeana Hook.f. & Euphorbiaceae & $\mathrm{H}$ & AVN/EUP/0192 \\
\hline 193. & Jatropha curcas $\mathrm{L}$. & Euphorbiaceae & $\mathrm{T}$ & AVN/EUP/0193 \\
\hline 194. & Euphorbia tithymaloides L. & Euphorbiaceae & $\mathrm{H}$ & AVN/EUP/0194 \\
\hline 195. & Phyllanthus virgatus G.Forst. & Euphorbiaceae & $\mathrm{H}$ & AVN/EUP/0195 \\
\hline 196. & Phyllanthus amarus Schumach. \& Thonn. & Euphorbiaceae & $\mathrm{H}$ & AVN/EUP/0196 \\
\hline 197. & Phyllanthus emblica L. & Euphorbiaceae & $\mathrm{T}$ & AVN/EUP/0197 \\
\hline 198. & Phyllanthus maderaspatensis $\mathrm{L}$. & Euphorbiaceae & $\mathrm{H}$ & AVN/EUP/0198 \\
\hline 199. & Sebastiania chamaelea (L.) Müll.Arg. & Euphorbiaceae & $\mathrm{H}$ & AVN/EUP/0199 \\
\hline 200. & Croton bonplandianus Baill. & Euphorbiaceae & $\mathrm{H}$ & AVN/EUP/0200 \\
\hline 201. & Artocarpus heterophyllus Lam. & Moraceae & $\mathrm{T}$ & AVN/MOR/0201 \\
\hline 202. & Ficus benghalensis $\mathrm{L}$. & Moraceae & $\mathrm{T}$ & AVN/MOR/0202 \\
\hline 203. & Ficus religiosa $\mathrm{L}$. & Moraceae & $\mathrm{T}$ & AVN/MOR/0203 \\
\hline 204. & Ficus benjamina L.Mant. var. benjamina & Moraceae & $\mathrm{T}$ & AVN/MOR/0204 \\
\hline 205. & Ficus carica $\mathrm{L}$. & Moraceae & $\mathrm{T}$ & AVN/MOR/0205 \\
\hline \multicolumn{5}{|c|}{ MONOCOTYLEDONS } \\
\hline 206. & Musa $\times$ paradisiaca $\mathrm{L}$. & Musaceae & $\mathrm{S}$ & AVN/MUS/400 \\
\hline 207. & Ravenala madagascariensis Sonn. & Sterlitziaceae & $\mathrm{S}$ & AVN/STE/0401 \\
\hline 208. & Canna indica $\mathrm{L}$. & Cannaceae & $\mathrm{H}$ & AVN/CAN/0402 \\
\hline 209. & Dracaena braunii Engl. & Agavaceae & $\mathrm{S}$ & AVN/AGA/0403 \\
\hline 210. & Aloe vera (L.) Burm.f. & Liliaceae & $\mathrm{H}$ & AVN/LIL/0404 \\
\hline 211. & Asparagus setaceus (Kunth) Jessop & Liliaceae & $\mathrm{S}$ & AVN/LIL/0405 \\
\hline 212. & Commelina benghalensis $\mathrm{L}$. & Commelinaceae & $\mathrm{H}$ & $\mathrm{AVN} / \mathrm{COM} / 0406$ \\
\hline 213. & Commelina ensifolia R.Br. & Commelinaceae & $\mathrm{H}$ & AVN/COM/0407 \\
\hline 214. & Commelina diffusa Burm.f. & Commelinaceae & $\mathrm{H}$ & $\mathrm{AVN} / \mathrm{COM} / 0408$ \\
\hline 215. & Borassus flabellifer $\mathrm{L}$. & Arecaceae & $\mathrm{T}$ & AVN/ARE/0407 \\
\hline 216. & Cocos nucifera $\mathrm{L}$. & Arecaceae & $\mathrm{T}$ & AVN/ARE/0408 \\
\hline 217. & Dypsis lutescens (H.Wendl.) Beentje \& J.Dransf. & Arecaceae & $\mathrm{S}$ & AVN/ARE/0409 \\
\hline 218. & Pandanus fascicularis Lam. & Pandanaceae & $\mathrm{S}$ & AVN/PAN/0410 \\
\hline 219. & Cyperus arenarius Retz. & Cyperaceae & $\mathrm{H}$ & AVN/CYP/0411 \\
\hline 220. & Cyperus rotundus L. & Cyperaceae & $\mathrm{H}$ & AVN/CYP/0412 \\
\hline 221. & Cyperus pangorei Rottb. & Cyperaceae & $\mathrm{H}$ & AVN/CYP/0413 \\
\hline 222. & Fimbristylis bisumbellata (Forssk.) Bubani & Cyperaceae & $\mathrm{H}$ & AVN/CYP/0414 \\
\hline 223. & Fimbristylis ferruginea (L.) Vahl & Cyperaceae & $\mathrm{H}$ & AVN/CYP/0415 \\
\hline 224. & Pycreus pumilus (L.) Nees & Cyperaceae & $\mathrm{H}$ & AVN/CYP/0416 \\
\hline 225. & Apluda mutica L. & Poaceae & $\mathrm{H}$ & AVN/POA/0417 \\
\hline 226. & Aristida adscensionis $\mathrm{L}$. & Poaceae & $\mathrm{H}$ & AVN/POA/0418 \\
\hline 227. & Aristida mutabilis Trin. \& Rupr. & Poaceae & $\mathrm{H}$ & AVN/POA/0419 \\
\hline 228. & Brachiaria reptans (L.) C.A.Gardner \& C.E.Hubb & Poaceae & $\mathrm{H}$ & AVN/POA/0420 \\
\hline 229. & Bracbiaria mutica (Forssk.) Stapf & Poaceae & $\mathrm{H}$ & AVN/POA/0421 \\
\hline 230. & Chloris barbata Sw. & Poaceae & $\mathrm{H}$ & AVN/POA/0422 \\
\hline 231. & Chrysopogon orientalis (Desv.) A.Camus & Poaceae & $\mathrm{H}$ & AVN/POA/0423 \\
\hline 232. & Cymbopogon citratus (DC.) Stapf & Poaceae & $\mathrm{H}$ & AVN/POA/0424 \\
\hline 233. & Cynodon dactylon (L.) Pers. & Poaceae & $\mathrm{H}$ & $\mathrm{AVN} / \mathrm{POA} / 0425$ \\
\hline 234. & Dactyloctenium aegyptium (L.) Willd. & Poaceae & $\mathrm{H}$ & AVN/POA/0426 \\
\hline 235. & Dichantbium annulatum (Forssk.) Stapf & Poaceae & $\mathrm{H}$ & AVN/POA/0427 \\
\hline 236. & Digitaria bicornis (Lam.) Roem. \& Schult. & Poaceae & $\mathrm{H}$ & AVN/POA/0428 \\
\hline 237. & Echinocbloa crus-galli (L.) P.Beauv. & Poaceae & $\mathrm{H}$ & AVN/POA/0429 \\
\hline 238. & Eragrostis amabilis (L.) Wight \& Arn. & Poaceae & $\mathrm{H}$ & AVN/POA/0430 \\
\hline 239. & Eragrostis minor Host & Poaceae & $\mathrm{H}$ & AVN/POA/0431 \\
\hline 240. & Eragrostis tenella (L.) P.Beauv. ex Roem. \& Schult. & Poaceae & $\mathrm{H}$ & AVN/POA/0432 \\
\hline 241. & Heteropogon contortus (L.) P.Beauv. ex Roem. \& Schult. & Poaceae & $\mathrm{H}$ & AVN/POA/0433 \\
\hline 242. & Imperata cylindrica (L.) Raeusch. & Poaceae & $\mathrm{H}$ & AVN/POA/0434 \\
\hline 243. & Iseilema laxum Hack. & Poaceae & $\mathrm{H}$ & AVN/POA/0435 \\
\hline 244. & Panicum psilopodium Trin. & Poaceae & $\mathrm{H}$ & AVN/POA/0436 \\
\hline 245. & Paspalidium flavidum (Retz.) A.Camus & Poaceae & $\mathrm{H}$ & AVN/POA/0437 \\
\hline 246. & Tragus roxburghii Panigrahi & Poaceae & $\mathrm{H}$ & AVN/POA/0438 \\
\hline 247. & Zoysia matrella (L.) Merr. & Poaceae & $\mathrm{H}$ & AVN/POA/0439 \\
\hline
\end{tabular}

Life form Abbreviations: H: Herb; S : Shrub ; T : Tree ; C : Climber 
Figure 2. Plant taxa documented in AV Campus
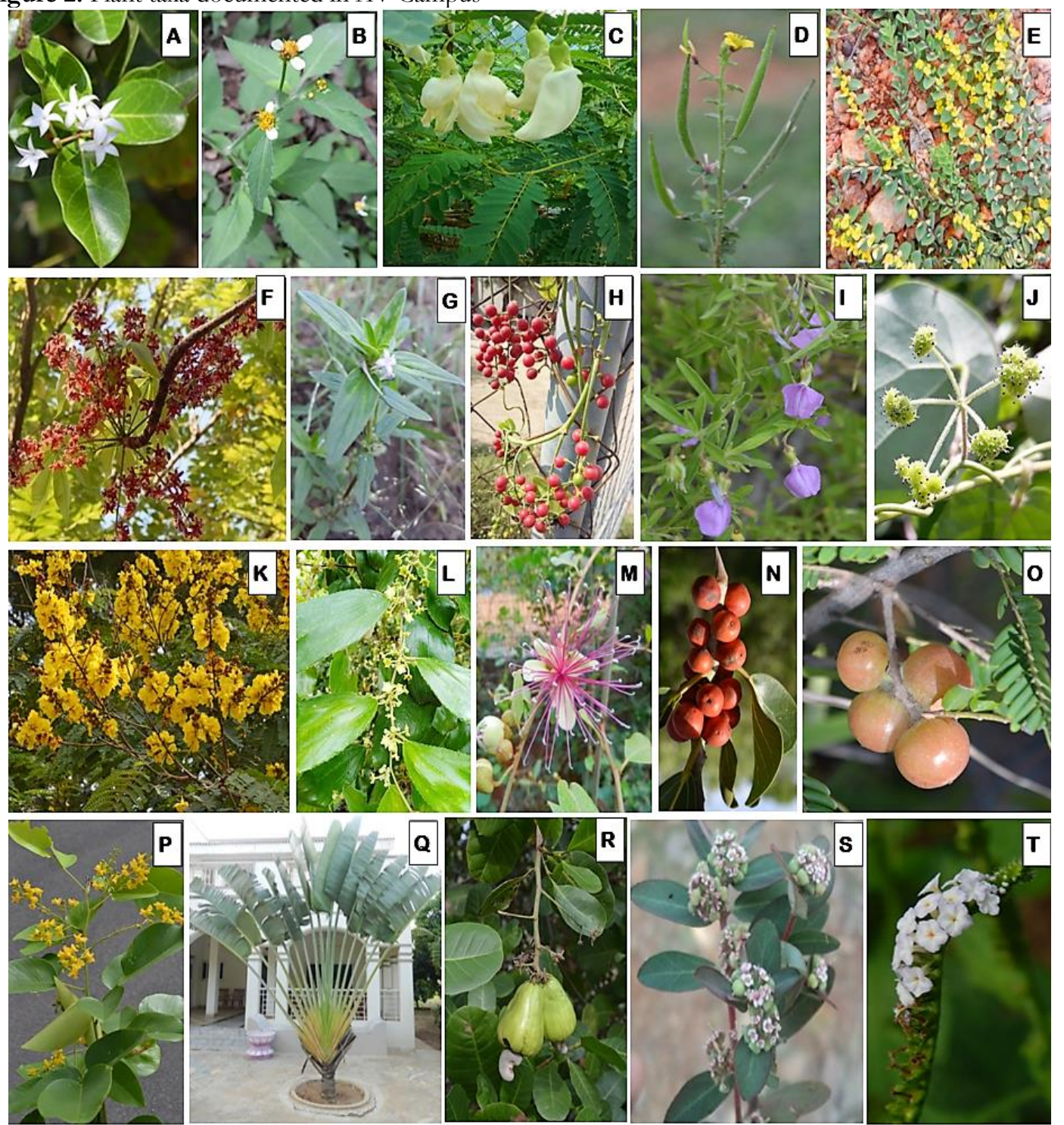

A: Carissa spinarum ; B: Bidens pilosa ; C: Sesbania grandiflora; D: Cleome viscosa ; $\mathbf{E}$ : Crotalaria hebecarpa; $\mathbf{F}$ : Sterculia foetida; $\mathbf{G}$ : Spermacoce aurticularis $; \mathbf{H}$ : Tinospora sinensis; $\mathbf{I}:$ Hybanthus enneaspermus $\mathbf{J}:$ Cyclea peltata $; \mathbf{K}$ : Peltophorum pterocarpum; L: Ziziphus jujuba; $\mathbf{M}$ :Capparis zeylanica; $\mathbf{N}$ : Ficus benghalensis; $\mathbf{O}$ : Phyllanthus emblica, $\mathbf{P}$ : Pterocarpus santalinus; $\mathbf{Q}$ : Ravenala madagascariensis; $\mathbf{R}:$ Anacardium occidentale; $\mathbf{S}:$ Euphorbia bypericifolia; $\mathbf{T}$ : Heliotropium indicum

\section{Summary and Conclusion}

This article deals with the list of 247 species of plant taxa occurring in the Akshara Vidyalaya (AV) campus which has a richlife form and diverse native, exotic and ornamental flora.

This floristic survey reveals that the native flora of AV Campus represents some medicinally important and economic important plants which has to be conserved in the garden to explore its medicinal importance to the students. Since the essentiality of documentation of campus flora and no other report on flora, this is the authentic report on the flora of campus of Akshara Vidyalaya. This type of curricular activity enhance an understanding and empathy with nature as a vital part of basic education in which Classroom theory is reinforced through practice in natural surroundings, permitting closer contact with the environment. We recommend the authorities and staff to introduce native and medicinal plant species to enrich the floral knowledge practically to the student community.

However, the present documentation is a baseline of the Plant taxa present and highlights the potential magnitude of biodiversity in the AV Campus. We expect that the information contained in the present study will be useful to the development of a management plan for any institution. 


\section{Acknowledgement}

The authors acknowledge the Authorities for the permission, financial support and necessary help to conduct this survey. A special gratitude to experts Ms. A. Lalithamba (Moderator, eFlora of IndiaGoogle group) and Mr. Mitta Mahendra nath (SRF, Department of Botany, Sri Venkateswara University) for identification of taxa and authors thankful to Mr. C.V. Saran, Mr. Nikhil Krishna and Mr. G. Vishnukanth Reddy for field work and documentation.

\section{References}

1. Gamble, J.S. and Fischer, C.E.C., Flora of the Presidency Madras. Vols. 1-3. Calcutta: Botanical Survey of India. 2017 pp. (Reprinted 1957; London: Adlard \& Co. London). (19151936).2017pp.

2. Pullaiah. T and Chennaiah. E. Flora of Andhra Pradesh(India).Ranunculaceae-Alangiaceae.

Scientific Publishers

(India), Jodhpur.(1997)Vol. I.print.

3. Pullaiah. T and Ali Moulali D. Flora of Andhra Pradesh(India). Rubiaceae - Ceratophyllaceae. Scientific Publishers (India), Jodhpur. (1997). Vol II. print.
4. Pullaiah. T Flora of Andhra Pradesh (India). Monocoyledons. Scientific Publishers (India), Jodhpur. (1997). Vol III. print.

5. Pullaiah, T. and P. Surya Prakash Babu Flora of Andhra Pradesh, India. Scientific Publishers, Jodhpur. (1998) Vol.4. print.

6. Suryanarayana, B. and Sreenivasa Rao A. (2001). Flora of Nellore district, Andhra Pradesh. Gurudev Prakashan, Srirampur (Maharastra). print.

7. The Plant List (2010). Ver 1. www.theplantlist.org.

8. Madhava Chetty, K., K. Sivaji \& K. Tulasi Rao Flowering Plants of Chittoor District, Andhra Pradesh, India. $4^{\text {th }}$ Edn. Students Offset Printers, Tirupati. (2015).600pp. print.

\section{Cite this article as:}

Manipal K., Kumuda M.V., Madhava Chetty K. Checklist of Akshara vidyalaya campus flora, Venkatachalam, Nellore, Andhra Pradesh, India. Annals of Plant Sciences 7.9 (2018) pp. 2423-2429. do http:/ / dx.doi.org/10.21746/aps.2018.7.9.4 